PROCEEDINGS OF THE

AMERICAN MATHEMATICAL SOCIETY

Volume 141, Number 7, July 2013, Pages 2515-2517

S 0002-9939(2013)11509-1

Article electronically published on February 28, 2013

\title{
GENERALIZED MINIMAX AND MAXIMIN INEQUALITIES FOR ORDER STATISTICS AND QUANTILE FUNCTIONS
}

\author{
JOEL E. COHEN \\ (Communicated by Walter Craig)
}

\begin{abstract}
Let $A$ be a finite real matrix with element $A(i, j)$ in row $i$ and column $j$. We generalize von Neumann's inequality $\min _{j} \max _{i} A(i, j) \geq$ $\max _{i} \min _{j} A(i, j)$ by replacing min by every order statistic.
\end{abstract}

\section{HISTORY}

Let $m$ and $n$ be finite positive integers, and let $A$ be a real $m \times n$ matrix with element $A(i, j)$ in row $i$ and column $j$. von Neumann [4] and Dresher [3, p. 13] proved that

$$
\min _{j} \max _{i} A(i, j) \geq \max _{i} \min _{j} A(i, j) .
$$

Equality holds in (1) if and only if for some (not necessarily unique) $i^{\prime}$ and $j^{\prime}, A\left(i^{\prime}, j^{\prime}\right)$ is the maximum of its column $j^{\prime}$ and the minimum of its row $i^{\prime}$.

For any real (row or column) vector $x$ with $l$ elements, and for any $1 \leq k \leq l$, let $\Lambda_{k}(x)$ denote the $k$ th smallest element of $x$ (the $k$ th order statistic of $x$ ). For example, $\Lambda_{1}(x)=\min (x), \Lambda_{l}(x)=\max (x)$, and for odd $l, \Lambda_{(l+1) / 2}(x)=\operatorname{median}(x)$. Let $A(i, \cdot)$ denote the $i$ th row and $A(\cdot, j)$ the $j$ th column of $A$. With this notation, (1) becomes

$$
\Lambda_{1}\left(\Lambda_{m}(A(\cdot, j))_{j=1}^{n}\right) \geq \Lambda_{m}\left(\Lambda_{1}(A(i, \cdot))_{i=1}^{m}\right) .
$$

We generalize this result by replacing the minimum with any $k$ th order statistic $\Lambda_{k}$.

Theorem. In the notation above, for any $1 \leq k \leq n$,

$$
\Lambda_{k}\left(\Lambda_{m}(A(\cdot, j))_{j=1}^{n}\right) \geq \Lambda_{m}\left(\Lambda_{k}(A(i, \cdot))_{i=1}^{m}\right) .
$$

If equality holds, then there exist $1 \leq i^{\prime} \leq m$ and $1 \leq j^{\prime} \leq n$ such that

$$
A\left(i^{\prime}, j^{\prime}\right)=\Lambda_{m}\left(A\left(\cdot, j^{\prime}\right)\right)=\Lambda_{k}\left(A\left(i^{\prime}, \cdot\right)\right) ;
$$

i.e., $A\left(i^{\prime}, j^{\prime}\right)$ equals a maximal element in column $j^{\prime}$ and the $k$ th order statistic of row $i^{\prime}$. Conversely, for $k=1$ and $k=n$, (3) implies (2). However, for $n>2$, if $1<k<n$, there exist $A, i^{\prime}$, and $j^{\prime}$ such that (3) holds, but strict inequality holds in (2). Also, for any $1 \leq k \leq n$,

$$
\Lambda_{k}\left(\Lambda_{1}(A(\cdot, j))_{j=1}^{n}\right) \leq \Lambda_{1}\left(\Lambda_{k}(A(i, \cdot))_{i=1}^{m}\right),
$$

with analogous conditions for equality.

Received by the editors June 14, 2011 and, in revised form, October 11, 2011.

2010 Mathematics Subject Classification. Primary 62C20, 62G30, 90C47, 91A05, 97K40.

Key words and phrases. Inequalities, minimax, maximin, order statistics, quantile functions.

(C)2013 Joel E. Cohen 
The theorem may be translated into equivalent inequalities for quantile functions.

Proof. For all $1 \leq p \leq m$ and $1 \leq q \leq n, A(p, q) \leq \Lambda_{m}(A(\cdot, q))$. Hence $A(p, \cdot) \leq$ $\Lambda_{m}(A(\cdot, j))_{j=1}^{n}$. (Inequality between vectors applies element by element.) It is well known (e.g., Davidson [2]) and elementary to prove that if $x$ and $y$ are vectors of the same length $l$ and $x \leq y$, then for every $1 \leq k \leq l, \Lambda_{k}(x) \leq \Lambda_{k}(y)$. Therefore $\Lambda_{k}(A(p, \cdot)) \leq \Lambda_{k}\left(\Lambda_{m}(A(\cdot, j))_{j=1}^{n}\right)$. This inequality holds for all $1 \leq p \leq m$, so (2) holds.

Now let $g=\Lambda_{m}\left(\Lambda_{k}(A(i, \cdot))_{i=1}^{m}\right)$ and $h=\Lambda_{k}\left(\Lambda_{m}(A(\cdot, j))_{j=1}^{n}\right.$ ) (we have $g \leq h$ by (2)) and suppose $g=h$. If every two elements of $A$ are distinct, there must exist $1 \leq i^{\prime} \leq m$ and $1 \leq j^{\prime} \leq n$ such that $g=h=A\left(i^{\prime}, j^{\prime}\right)$. Since $g$ is the maximum over rows of the $k$ th order statistic of each row, element $g=A\left(i^{\prime}, j^{\prime}\right)$ is, in particular, the $k$ th order statistic of row $i^{\prime}$. Since $h$ is the $k$ th smallest of the column maxima, element $h=A\left(i^{\prime}, j^{\prime}\right)$ is, in particular, the maximum of column $j^{\prime}$. Hence (3) holds.

If $g=h$ and at least one element of $A$ equals at least one other element of $A$, let $g=A\left(i^{\prime}, j^{\prime}\right)$ for some $i^{\prime}$ and some $j^{\prime}$. If the number of elements of $A$ equal to $g$ equals 1 , then again (3) holds by the previous argument. If two or more elements of $A$ equal $g$, then $h=A\left(p^{\prime}, q^{\prime}\right)$ with $p^{\prime} \neq i^{\prime}$ or $q^{\prime} \neq j^{\prime}$ is possible. But $g=h$ implies that $A\left(i^{\prime}, j^{\prime}\right)=A\left(p^{\prime}, q^{\prime}\right)$. By the previous argument, (3) holds.

Establishing whether (3) implies equality in (2) involves three cases: $k=1$, $k=n$, and $1<k<n$. Assume (3).

If $k=1$, then $A\left(i^{\prime}, j^{\prime}\right)=\Lambda_{1}\left(A\left(i^{\prime}, \cdot\right)\right) \leq \Lambda_{m}\left(\Lambda_{1}(A(i, \cdot))_{i=1}^{m}\right)$ and $A\left(i^{\prime}, j^{\prime}\right)=$ $\Lambda_{m}\left(A\left(\cdot, j^{\prime}\right)\right) \geq \Lambda_{1}\left(\Lambda_{m}(A(\cdot, j))_{j=1}^{n}\right)$, whence $\Lambda_{1}\left(\Lambda_{m}(A(\cdot, j))_{j=1}^{n}\right) \leq \Lambda_{m}\left(\Lambda_{1}(A(i, \cdot))_{i=1}^{m}\right)$. But for $k=1$, (2) becomes $\Lambda_{1}\left(\Lambda_{m}(A(\cdot, j))_{j=1}^{n}\right) \geq \Lambda_{m}\left(\Lambda_{1}(A(i, \cdot))_{i=1}^{m}\right)$. Hence equality holds in (2). If $k=n$, (2) becomes $\Lambda_{m}\left(\Lambda_{m}(A(\cdot, j))_{j=1}^{n}\right) \geq \Lambda_{m}\left(\Lambda_{m}(A(i, \cdot))_{i=1}^{m}\right)$. Here equality holds trivially because both sides refer to the maximal element(s) of matrix $A$.

When $1<k<n$, let

$$
A=\left(\begin{array}{lll}
1 & 2 & 5 \\
6 & 4 & 3
\end{array}\right) .
$$

Then the column maxima of $A$ are $\Lambda_{2}(A(\cdot, j))_{j=1}^{3}=(6,4,5)$, and the vector of order statistics is $\Lambda\left(\Lambda_{2}(A(\cdot, j))_{j=1}^{3}\right)=(4,5,6)$, while the maxima of the ordered rows are

$$
\Lambda_{2}\left(\Lambda\left(A(i, \cdot)_{i=1}^{2}\right)\right)=\Lambda_{2}\left(\begin{array}{lll}
1 & 2 & 5 \\
3 & 4 & 6
\end{array}\right)=(3,4,6) .
$$

Hence, as required by $(2), \Lambda\left(\Lambda_{2}(A(\cdot, j))_{j=1}^{3}\right)=(4,5,6) \geq \Lambda_{2}\left(\Lambda\left(A(i, \cdot)_{i=1}^{2}\right)\right)=$ $(3,4,6)$. Element $A(2,2)=4$ is the maximum element in its column and the second order statistic $(k=2)$ in its row, but $\Lambda_{2}\left(\Lambda_{2}(A(\cdot, j))_{j=1}^{3}\right)=5>\Lambda_{2}\left(\Lambda_{2}(A(i, \cdot))_{i=1}^{2}\right)=$ 4 , so equality does not hold in (2) when $k=2$.

To prove (4), observe that for vector $x$ as above and for any $1 \leq k \leq l, \Lambda_{k}(x)=$ $-\Lambda_{l+1-k}(-x)$. Therefore (using (2) multiplied by -1 for the inequality step below)

$$
\begin{aligned}
& \Lambda_{k}\left(\Lambda_{1}(A(\cdot, j))_{j=1}^{n}\right)=-\Lambda_{n+1-k}\left(-\Lambda_{1}(A(\cdot, j))_{j=1}^{n}\right)=-\Lambda_{n+1-k}\left(\Lambda_{m}(-A(\cdot, j))_{j=1}^{n}\right) \\
& \leq-\Lambda_{m}\left(\Lambda_{n+1-k}(-A(i, \cdot))_{i=1}^{m}\right)=-\Lambda_{m}\left(-\Lambda_{k}(A(i, \cdot))_{i=1}^{m}\right)=\Lambda_{1}\left(\Lambda_{k}(A(i, \cdot))_{i=1}^{m}\right) .
\end{aligned}
$$

The special case of a $2 \times 2$ matrix is easy to analyze completely. The numbers $1,2,3,4$ may be entered in a $2 \times 2$ matrix in $4 !=24$ ways. Because the order statistics of rows and columns are invariant under exchanges of rows and exchanges of columns, there are only $4 ! /(2 ! 2 !)=6$ distinct cases to consider. We may select those 6 cases by setting $A(1,1)=1$ and filling in the remaining elements with 
all permutations of 2,3,4. Equality in (2) for $k=1,2$ holds in four cases. An inequality arises in (2) if

$$
A=\left(\begin{array}{ll}
1 & 3 \\
4 & 2
\end{array}\right) \text { or } A=\left(\begin{array}{ll}
1 & 4 \\
3 & 2
\end{array}\right)
$$

In these cases, $\Lambda\left(\Lambda_{2}(A(\cdot, j))_{j=1}^{2}\right)=(3,4)$, while $\Lambda_{2}\left(\Lambda\left(A(i, \cdot)_{i=1}^{2}\right)\right)=(2,4)$.

\section{INTERPRETATIONS}

The theorem is relevant to applied problems in bootstrap sampling [1, industrial life testing, and the economic analysis of poverty. In bootstrap sampling, one computes a statistic $A(i, j)$ for each case $i$ and each sample $j$, obtaining an $m \times n$ matrix $A$. A central tendency of the maximal value of $A(i, j)$ could be estimated as the maximum over cases $i$ of the median of $A(i, \cdot)$ or as the median over samples $j$ of the maximum of $A(\cdot, j)$. The theorem shows that the second procedure gives the (weakly) larger value. In life testing, one observes the life lengths $A(i, j)$ of $n$ machines labeled $j$ under $m$ conditions labeled $i$. In the economics of poverty, one observes the incomes of $n$ people labeled $j$ in $m$ localities labeled $i$. Order statistics of the minimal (respectively, maximal) life length (or income) may be estimated from the expressions on the left and right sides of (4) (resp., (2)), and the theorem relates these estimates.

\section{ACKNOWLEDGMENTS}

Suggestions from editor Walter Craig and two anonymous referees greatly improved the paper. The author acknowledges, with thanks, the support of U.S. National Science Foundation grant EF-1038337, the assistance of Priscilla K. Rogerson, and the hospitality of the family of William T. Golden.

\section{REFERENCES}

[1] Joel E. Cohen and Jacob Oppenheim, Is a limit to the median length of human life imminent? Genus 68(1):11-40, 2012.

[2] Russell Davidson, Stochastic dominance. The New Palgrave Dictionary of Economics, eds. Steven N. Durlauf and Lawrence E. Blume, Palgrave Macmillan, 2008.

[3] Melvin Dresher, Games of Strategy: Theory and Applications. Prentice-Hall, Englewood Cliffs, 1961. Reprinted as: The Mathematics of Games of Strategy: Theory and Applications. Dover, New York, 1981. MR0122586 (22:13310) MR.0671740 (83m:90093)

[4] J. von Neumann, Zur Theorie der Gesellschaftsspiele, Mathematische Annalen 100, 295-320, 1928. Translated as "On the Theory of Games of Strategy", in Contributions to the Theory of Games, Volume IV, pp. 13-42 (Annals of Mathematics Studies, 40) (A. W. Tucker and R. D. Luce, eds.), Princeton University Press, Princeton, 1959. MR.1512486 MR.0101828 $(21: 635)$

Laboratory of Populations, The Rockefeller University and Columbia University, 1230 York Avenue, New York, New York 10065

E-mail address: cohen@rockefeller.edu 